\title{
Valuing An Emerging International Technology Company
}

John Zimmerman, Zayed University, UAE

\begin{abstract}
The requirements of Financial Accounting Standard Board (FASB) 142 provide an excellent opportunity to examine various financial valuation methods used to determine a company's value. Under FASB 142, goodwill and intangible assets with indefinite useful lives are no longer amortized, but instead tested for impairment at least annually in accordance with the provisions. Any impairment loss has to be measured as of the date of adoption and recognized as the cumulative effect of a change in accounting principle in an organization's first interim period. The impairment test requires an accurate and fair valuation of the asset in question. This case is based upon the valuation dilemma faced by Integrated Silicon Solution (NASDAQ: ISSI), a publicly traded international technology company, in late 2008. ISSI had made several acquisitions and carried substantial goodwill. Since ISSI was publicly traded, a public market value was available but the financial crisis of 2008 caused the company to consider other methods, as is allowed under FASB 142. The case uses both the income and comparable market approaches to arrive at a fair value, and this value is used to determine if impairment for the goodwill the company carried on its balance sheet existed.
\end{abstract}

Keywords: FASB 142; income valuation approach; comparable company valuation approach; impairment; fair value; goodwill; intangible assets

\section{INTRODUCTION}

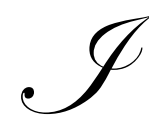

n September, 2008 Paula Zebrowski, the corporate controller for Integrated Silicon Solution (NASDAQ: ISSI), faced a serious issue. The stock market was awful, the NASDAQ had dropped substantially due to the financial crisis, and ISSI, a fabless semiconductor company that designs and markets high speed and low power static random access memory ("SRAM") as well as low and medium density dynamic random access memory ("DRAM") integrated circuits primarily for industrial, automotive, and telecommunications applications, had its stock price hammered like everyone else.

Paula knew that the goodwill ISSI carried on its balance sheet from some acquisitions made over the years must be evaluated for impairment consistent with the requirements of Financial Accounting Standard Board (FASB) 142. Normally this was a simple task. ISSI was a publically traded company, so quoted market prices in active markets were available, and she knew that this value is normally the best evidence of fair value. However, given current market conditions, she suspected the ISSI current market price may not be representative of the fair value of the company. In reviewing FASB 142 she had noted that the standard stated that the quoted market price of an individual equity security need not be the sole measurement basis of the fair value of a reporting unit, and if fact, may not be the best valuation to use.

Therein was the dilemma facing the company, which techniques should the company use to fairly value the enterprise, what valuation was most representative, and was there a FASB 142 impairment? Paula decided that she needed an independent analysis and so she engaged a consulting firm to assist her in her analysis. 


\section{ISSI BACKGROUND ${ }^{1}$}

ISSI (the Company) was incorporated in 1988 and completed its initial public offering in 1995. The Company designs, develops, and markets high performance integrated circuits ("ICs") for the digital consumer electronics, networking, mobile communications, automotive electronics, and industrial/medical electronics markets. The Company's primary offering includes high speed and low power static random access memory ("SRAM") as well as low and medium density dynamic random access memory ("DRAM"). Additionally, the Company designs and markets electrically erasable programmable read only memory ("EEPROM") and Smartcards, along with developing selected non-memory products focused on its primary markets.

The Company outsources the manufacture of its products based upon joint technology relationships with foundries in Asia as well as the United States. Additionally, the Company makes equity investments in various foundries on a global basis. The Company has added design groups in Shanghai, China and Hsinchu, Taiwan, complementing a core engineering and product management team located in San Jose and Santa Clara, California. Finally, the Company has made a number of business purchases in order to further grow its business. In February 2002, ISSI acquired Purple Ray, Inc. ("Purple Ray") in order to increase its manufacturing and development capabilities to produce ICs that enable network systems to retrieve data bytes on a simultaneous basis. In December 2004, ISSI acquired a stake in Signia Technologies, Inc. ("Signia") in order to diversify into non-memory chip products and to strengthen its strategic relationship with Signia. Finally, in August 2005, ISSI acquired a majority stake in Integrated Circuit Solution, Inc. ("ICS") in order to scale purchasing power, enhance product offerings, gain new customers and improve economies of scale and operating efficiencies.

The Company's customers include leaders in the target markets, including Apex, Samsung, Sony, 3Com, Cisco, Yahoo!, LG Electronics, Motorola, Bose, Siemens, General Electric, and Tyco, among others. These relationships, along with the increasing demand for high performance memory devices across a wide range of end markets, provide significant opportunities for ISSI to expand its high performance IC business. ISSI is headquartered in San Jose, California, with additional offices in China, Europe, Hong Kong, India, Japan, Korea, Singapore, Taiwan and the United States. In September, 2008, the Company employed approximately 500 individuals, the majority of which were located in Taiwan.

\section{THE INDUSTRY}

The supply-demand nexus for portable electronic devices such as MP3 players and portable gaming devices is driving the consumer electronics market. Given the slowing economic environment, the growth of spending in consumer electronics is declining on a global basis. The Consumer Electronics Association (CEA) has taken into account global economic conditions and projects the 2008 global market for consumer electronics to equal $\$ 618.0$ billion in retail with the U.S. accounting for approximately $\$ 148.3$ billion or $24 \%$ of the worldwide total ${ }^{2}$. Given that a key component in consumer electronics is the IC, the growth of the semiconductor industry is expected to mirror that of the consumer electronics market. Based on the latest iSuppli Corporation ("iSuppli") figures, worldwide sales of semiconductors are expected to grow from $\$ 260.2$ billion in 2006 to $\$ 291.4$ billion by 2008 , which implies a $5.8 \%$ compound annual growth rate ("CAGR") from 2006 to $2008^{3}$. Semiconductor market conditions are expected to be weak throughout 2008 due to a variety of factors such as oversupply, declining prices and rising energy costs. One study indicated that growth was projected at $3.4 \%$ in 2008, with the DRAM market providing the major drag on sales ${ }^{4}$ Despite these decreasing projections, a separate study indicated that long run IC unit shipments are expected to increase at least 10\% annually over the next 5-10 years, as new and evolving applications in communications and consumer electronic systems continue to incorporate large quantities of advanced ICs ${ }^{5}$.

\footnotetext{
${ }^{1}$ This case is based upon work completed by the author, the company, and by Financial Strategies Consulting, LLC.

${ }^{2}$ Consumer Electronics Association 2008 Outlook. (2008). Retrieved June, 20, 2008 from www.ce.org.

${ }^{3}$ iSuppli Corporation. 2007 and 2008 Semiconductor Forecast Adjustments (Fact Sheet). El Segundo, CA: iSuppli Corporation.

${ }^{4}$ The Gartner Group. (2008, March 3, 2008). Gartner halves 2008 semiconductor market growth estimate. Electronic News.

${ }^{5}$ IC Insights. (2007). The McClean Report. Retrieved July 25, 2007 from www.icinsights.com
} 


\section{GLOBAL ECONOMIC CONDITIONS}

As part of the analysis, the company analyzed the global economy as well as the economies of the U.S., Asia and the Euro-Zone. In determining the outlook of these economies, gross domestic product ("GDP"), the broadest gauge of total economic performance, was reviewed. The basic data for the analysis was derived from information provided by the International Monetary Fund ${ }^{6}$.

\section{WORLD ECONOMY}

According to the IMF, world economic output growth equaled 5.1\% in 2006, decreasing to $5.0 \%$ in 2007 due to the financial market crisis that erupted during the second half of 2007. The turmoil began in the U.S. amid awareness of rising defaults on sub-prime mortgages resulting from previous credit market excesses and a subsequent housing correction. The rising defaults also affected many international banks that were exposed to U.S. sub-prime backed fixed income securities. Consequently, the fallout curtailed liquidity in the interbank market, weakened the balance sheets at major banks and prompted significant re-pricing of risk across a broad range of financial instruments. These actions have caused business and consumer sentiment to retreat, industrial production to weaken and business activity to stall through the first half of calendar year 2008. To compound problems, inflation continues to rise due to high commodity and food prices. In particular, oil prices have become a primary concern as news signaling risks of short-term supply disruptions, including those related to geopolitical risks, have propelled oil barrel costs to record levels. Against this background, the IMF projected economic output growth to decelerate to $4.1 \%$ and $3.9 \%$ in 2008 and 2009, respectively. Specific data concerning GDP growth rates for Asia, Europe, and the US depicted similar patterns.

\section{FASB 142}

On June 29, 2001, the FASB approved for issuance FASB 142, "Goodwill and Other Intangible Assets"7 This statement was finalized and issued on July 20, 2001 and has changed the accounting for goodwill from an amortization method to an impairment-only approach.

Under FASB 142, goodwill and intangible assets with indefinite useful lives are no longer amortized, but instead tested for impairment at least annually in accordance with the provisions of FASB 142. Any impairment loss has to be measured as of the date of adoption and recognized as the cumulative effect of a change in accounting principle in an organization's first interim period.

The first step of the goodwill impairment test, used to identify potential impairment, compares the fair value of a reporting unit with its carrying amount, including goodwill. If the fair value of a reporting unit exceeds its carrying amount, goodwill of the reporting unit is considered not impaired, thus the second step of the impairment test is unnecessary. If the carrying amount of a reporting unit exceeds its fair value, the second step of the goodwill impairment test shall be performed to measure the amount of impairment loss, if any.

The second step of the goodwill impairment test used to measure the amount of impairment loss by comparing the implied fair value of reporting unit goodwill with the carrying amount of that goodwill. If the carrying amount of reporting unit goodwill exceeds the implied fair value of that goodwill, an impairment loss shall be recognized in an amount equal to that excess. The loss recognized cannot exceed the carrying amount of goodwill. After a goodwill impairment is recognized, the adjusted carrying amount of goodwill shall be its new accounting basis. Subsequent reversal of a previously recognized goodwill impairment loss is prohibited once the measurement of that loss is completed.

\footnotetext{
${ }^{6}$ International Monetary Fund. World economic outlook update: global slowdown and rising inflation. Retrieved July 30,2008 from www.imf.org

${ }^{7}$ Summary of FASB 142. (2001). Retrieved August 15, 2010, from www.fasb.org
} 


\section{SCOPE OF ANALYSIS}

Paula and the consultants worked closely with ISSI management to complete a cash flow analysis of the associated long-term intangible assets in accordance with FASB 142. The steps to be undertaken included:

1) Interviewing senior management at ISSI regarding the status and business prospects of the Company.

2) Discussing with ISSI's management the business prospects of the Company.

3) Analyzing certain historical financial statements as well as certain financial forecasts and other data provided to us by ISSI's management. Reviewing assumptions, including growth rates and operating margins and discussing the rationale of these assumptions with management.

4) Completing a detailed cash flow analysis of the Company.

The results of this analysis are depicted in the table below, and in the Appendices.

\begin{tabular}{|l|l|}
\hline \multicolumn{1}{|c|}{ Item } & \multicolumn{1}{|c|}{ Comment } \\
\hline Intangible Assets at September 30, 2008 & $\begin{array}{l}\text { \$27.4 million resulting from various acquisitions. \$2.1 million was } \\
\text { identified as identifiable/amortizable intangibles, and \$25.3 } \\
\text { million consisted of goodwill. }\end{array}$ \\
\hline Reporting Basis & ISSI is consolidated into one reporting unit. \\
\hline 2008 Balance Sheet & Appendix A-0 \\
\hline 2009 through 2013 Five Year Financial Projections & Appendix A-1 \\
\hline $\begin{array}{l}\text { Working Capital, Depreciation/Amortization, and Capital } \\
\text { Expenditure Assumptions }\end{array}$ & Appendix A-2 \\
\hline Net Operating Losses Carryforward Analysis & Appendix A-3 \\
\hline Comparable Companies & Appendix B-0 \\
\hline Comparable Companies & Appendix B-1 \\
\hline Weighted Average Cost of Capital & Appendix B-2 \\
\hline
\end{tabular}

This data forms the basis for addressing the following questions:

1) Using a Market Approach and Income Approach, what is your recommendation for a fair market value for ISSI at September 30, 2008?

2) Complete a detailed impairment assessment. What would be your recommendation to the chief financial and chief executive officers regarding FASB 142 ? 
Appendix A-0

2008 Balance Sheet ${ }^{8}$

Balance Sheet

Assets

Current Assets

Cash \& Equivalents

Accounts Receivable

Inventories

Other Current Assets

Net Property Plant \& Equipment

Long-term Investments

Other Assets

Intangible Assets

Goodwill

Other Acquisition Intangibles

\section{Total Assets}

\section{Liabilities}

Current Liabilities

Current Debt

Accounts Payable

Accrued Compensation and Benefits

Accrued Expenses

Other Long-Term Liabilities

Total Liabilities

Minority Interest

Stockholders' Equity

Total Stockholders' Equity

Total Liabilities \& Stockholders' Equity

\section{$8 / 31 / 2008$}

$$
\begin{array}{r}
\$ 55,962,396 \\
27,773,829 \\
47,837,325 \\
4,321,355 \\
\hline 135,894,905
\end{array}
$$

$24,202,000$

$18,882,323$

$1,437,629$

$25,338,402$

2068717

$27,407,119$

$207,823,976$

317,259

$37,658,737$

$3,308,330$

$7,541,645$

$48,825,971$

577,863

$49,403,834$

723,276

$157,696,866$

$207,823,976$

Appendix A-1

Projected Income Statement/Other Assumptions

FY $2009-2013^{9}$

\begin{tabular}{|l|c|c|c|c|c|}
\hline \multicolumn{1}{|c|}{$\mathbf{\$ 0 0 0}$ 's } & $\mathbf{9 / 3 0 / 2 0 0 9}$ & $\mathbf{9 / 3 0 / 2 0 1 0}$ & $\mathbf{9 / 3 0 / 2 0 1 1}$ & $\mathbf{9 / 3 0 / 2 0 1 2}$ & $\mathbf{9 / 3 0 / 2 0 1 3}$ \\
\hline Revenue & 249,400 & 290,000 & 335,000 & 380,000 & 420,000 \\
\hline COGS & 192,000 & 221,800 & 252,900 & 285,000 & 310,800 \\
\hline Gross Profit & 57,400 & 68,200 & 82,100 & 95,000 & 109,200 \\
\hline Operating Expenses & 53,200 & 57,000 & 61,500 & 67,500 & 73,500 \\
\hline Operating Income & 4,200 & 11,200 & 20,600 & 27,500 & 35,700 \\
\hline Taxes & 1,680 & 4.480 & 8,240 & 11,000 & 14,280 \\
\hline Net Income & 2,520 & 6,720 & 12,360 & 16,500 & 21,420 \\
\hline
\end{tabular}

${ }^{8}$ Provided by ISSI management

${ }^{9}$ Provided by ISSI Management 


\section{Appendix A-1}

Working Capital, Depreciation/Amortization, And Capital Expenditures Assumptions ${ }^{10}$

Assumptions:

1) Working Capital as a percentage of revenue $-15 \%$

2) Capital Expenditures and Depreciation/Amortization:

\begin{tabular}{|c|c|c|c|c|c|c|c|}
\hline \multirow[b]{2}{*}{$\$ 000$ 's } & \multirow{2}{*}{\multicolumn{2}{|c|}{$\begin{array}{c}\text { Capital } \\
\text { Expenditures (1) }\end{array}$}} & \multicolumn{5}{|c|}{ Depreciation/Amortization Schedule (2) } \\
\hline & & & $9 / 30 / 2009$ & $9 / 30 / 2010$ & 9/30/2011 & $9 / 30 / 2012$ & $9 / 30 / 2013$ \\
\hline $9 / 30 / 2009$ & $\$$ & $3,000.0$ & 350.0 & 700.0 & 700.0 & 575.0 & 450.0 \\
\hline $9 / 30 / 2010$ & $\$$ & $3,500.0$ & & 408.0 & 817.0 & 817.0 & 671.0 \\
\hline $9 / 30 / 2011$ & $\$$ & $4,000.0$ & & & 467.0 & 933.0 & 933.0 \\
\hline $9 / 30 / 2012$ & $\$$ & $4,500.0$ & & & & 525.0 & $\$ 1,050.0$ \\
\hline $9 / 30 / 2013$ & $\$$ & $5,000.0$ & & & & & 583.0 \\
\hline \multicolumn{3}{|c|}{ Depreciation of existing assets (3) } & $\$ 4,840.0$ & $\$ 4,840.0$ & $\$ 4,840.0$ & $\$ 4,840.0$ & $\$ 4,840.0$ \\
\hline \multicolumn{3}{|c|}{ Total Dereciation/Amortization } & $\$ 5,190.0$ & $\$ 5,948.0$ & $\$ 6,824.0$ & $\$ 7,690.0$ & $\$ 8,527.0$ \\
\hline \multicolumn{8}{|c|}{ (1) Capital expeditures based upon historical percentages of sales. } \\
\hline \multicolumn{8}{|c|}{ (2) Assumes straight line deprceiation over either 3 or 5 years. } \\
\hline
\end{tabular}

Appendix A-2

Value of Net Operating Losses Carryforward ${ }^{11}$

\begin{tabular}{|c|c|c|c|c|c|c|c|c|c|c|c|}
\hline \multicolumn{4}{|c|}{ Present Value Of NOLs (\$000s) } & \multirow{2}{*}{\multicolumn{2}{|c|}{$9 / 30 / 2010$}} & & & 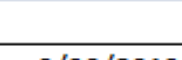 & \\
\hline & & & $9 / 30 / 2009$ & & & & $9 / 30 / 2011$ & & /30/2012 & $9 / 30 / 2013$ & $9 / 30 / 2014$ \\
\hline Available NOLs (1) & & $\$$ & $121,700.0$ & & & & & & & & \\
\hline Operating Income & & $\$$ & $4,200.0$ & $\$$ & $11,200.0$ & $\$$ & $20,600.0$ & $\$ 2$ & $27,500.0$ & $\$ 35,700.0$ & \\
\hline Beginning Balance & & & $121,700.0$ & & $117,500.0$ & $\$$ & $106,300.0$ & $\$ \varepsilon$ & $85,700.0$ & $\$ 58,200.0$ & $\$ 22,500.0$ \\
\hline NOL Utilized & & $\$$ & $4,200.0$ & $\$$ & $11,200.0$ & $\$$ & $20,600.0$ & $\$ 2$ & $27,500.0$ & $\$ 35,700.0$ & $\$ 22,500.0$ \\
\hline Tax Benefit (2) & $35 \%$ & $\$$ & $1,470.0$ & $\$$ & $3,920.0$ & $\$$ & $7,210.0$ & $\$$ & $9,625.0$ & $\$ 12,495.0$ & $\$ 7,875.0$ \\
\hline PV Factor & $24 \%$ & & 0.8980 & & 0.7242 & & 0.5840 & & 0.4710 & 0.3798 & 0.3063 \\
\hline $\mathrm{PV}$ of NOL & & $\$$ & $1,320.1$ & $\$$ & $2,838.9$ & $\$$ & $4,210.6$ & $\$$ & $4,533.4$ & $\$ 4,745.6$ & $\$ \quad 2,412.1$ \\
\hline TotalPV of NOLs & & & $20,060.7$ & & & & & & & & \\
\hline & L) Per 200 & 7 & nancial stat & tem & ents & & & & & & \\
\hline & 2) US Fed & eral & Tax Rate & & & & & & & & \\
\hline
\end{tabular}

\footnotetext{
${ }^{10}$ Provided by ISSI Management

${ }^{11}$ Provided by ISSI Management
} 


\section{Appendix B-0 \\ Comparable Companies ${ }^{12}$}

\section{D西:}

- Designs, develops, manufactures, and markets a range of semiconductor IC products in the U.S., Asia, and Europe. The company's products include microcontrollers, logic, mixed-signal, nonvolatile memory and RF components.

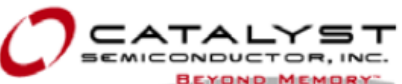

ветоnо memore - Engages in the design, development, and marketing of reprogrammable nonvolatile memory products and analog/mixed-signal semiconductor products. Products include EEPROM and flash memory products, which are used in applications to store user reconfigurable data in a wide variety of consumer electronics, as well as analog and mixed-signal products comprising supervisory products with EEPROM.

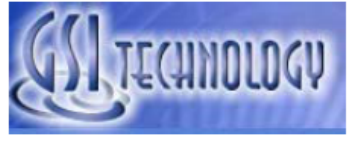

- Designs, develops, and markets SRAM products for the networking and telecommunications markets. Its SRAM products are incorporated in high-performance equipment, such as routers, switches and base stations. The company offers synchronous SRAM products that are controlled by timing signals, referred to as clocks; and asynchronous SRAM products, which employ a clock-free control interface.

\section{DIDI} communications, computing, and consumer industries worldwide. The company operates in three segments: Networking, Timing and Memory Interface, and Standard Products and Other. The Networking segment offers network search engines and search accelerators, switching solutions for server and storage applications, memories to solve inter-chip communications problems, multi-port memory products, and integrated communications processors. The Timing and Memory Interface segment provides products for clock generation, distribution, recovery, and jitter attenuation, memory interface products, consumer clocks for digital consumer applications, and communication clocks. The Standard Products and Other segment offers telecommunications semiconductor products, including products for access and transport, time-division multiplex switching, and voice processing; digital logic products for network switches, routers, wireless base stations, and storage networks, SRAMs for the communications and other markets, data conversion products, custom application specific integrated circuits, and multi-chip modules for military/aerospace applications, and mixed signal technology for video interfaces in digital video equipment.

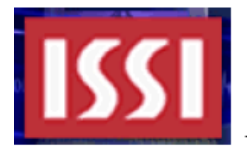

- Designs and markets integrated circuits for digital consumer electronics, networking, mobile communications, and automotive electronics markets. Company products include high speed and low power SRAM and low and medium density DRAM. It also designs and markets EEPROMs, SmartCards, and non-memory products.

\footnotetext{
${ }^{12}$ Information for Appendices B-0 and B-1 were obtained from company websites.
} 


\section{Appendix B-0 \\ Comparable Companies ${ }^{13}$ \\ (continued)}

\section{Dímicrochip} products for embedded control applications. Its product portfolio comprises 8-bit, 16-bit, and 32-bit microcontrollers, and 16-bit digital signal controllers, which features on-board flash memory technology. The company also provides development tools; analog and interface products that consist of power management, linear, mixed-signal, thermal management, and interface products; and various memory products.

\section{Micron}

- Engages in the manufacture and marketing of semiconductor devices worldwide. Its products include DRAM products that provide data storage and retrieval. The company also offers not-and ("NAND") flash memory products, which are electrically re-writeable and non-volatile semiconductor devices that retain memory content when power is turned off; and complementary metal-oxide semiconductor image sensors that capture and process images into pictures or video for various consumer and industrial applications. The company serves original equipment manufacturers, retailers, and distributors.

\section{Sill SiliciconMotion}

- Designs, develops, markets, and supplies semiconductor solutions for the multimedia consumer electronics market. The company offers multimedia system-on-a-chip products for various consumer products and storage applications, as well as mobile communications products. The company sells its semiconductor solutions to original equipment manufacturers and original design manufacturers.

$\boldsymbol{A P C H} \boldsymbol{H}$ - Designs and manufactures memory products with three main business groups: DRAM Product Business Group, Memory IC Manufacturing Business Group, and Flash Memory IC Business Group. The DRAM products are focused on the mobile memory market. The company's Low Power DRAM is designed to meet the need of portable multimedia devices for high-performance memory with low power consumption.

\footnotetext{
${ }^{13}$ Information for Appendices B-0 and B-1 were obtained from company websites.
} 


\begin{tabular}{|c|c|c|c|c|c|c|c|c|c|c|c|c|c|c|}
\hline \multirow[b]{2}{*}{$\$ 000$ 's } & \multicolumn{9}{|c|}{ Appendix B-1 Comparable Companies Financial Analysis } & \multirow{2}{*}{\multicolumn{2}{|c|}{ MU }} & \multirow[b]{2}{*}{ SIMO } & \multirow{2}{*}{\multicolumn{2}{|c|}{ 2344.TW }} \\
\hline & \multicolumn{2}{|r|}{ ATML } & \multicolumn{2}{|r|}{ CATS } & GSIT & \multicolumn{2}{|r|}{ IDTI } & ISSI & $\mathrm{MCHP}$ & & & & & \\
\hline Est. FY08 Revenue & $\$$ & $1,680,000$ & $\$$ & 75,300 & $\$ 68,350$ & $\$$ & 795,420 & $\$ 238,560$ & $\$ 1,090,000$ & $\$$ & $5,940,000$ & $\$ 197,740$ & & \\
\hline \%Change vs. Last FY & & 2.49 & & -0.83 & 28.65 & & 1.79 & -2.79 & 5.24 & & 4.43 & 11.09 & & \\
\hline Est. FY09 Revenue & $\$$ & $1,780,000$ & $\$$ & 78,150 & $\$ 73,040$ & $\$$ & 858,140 & $\$ 245,680$ & $\$ 1,190,000$ & $\$$ & 660,000 & $\$ 224,920$ & & \\
\hline \%Change vs. Last FY & & 4.21 & & 1.45 & 17.21 & & 4.79 & 0.06 & 7.19 & & 6.21 & 12.41 & & \\
\hline Revenue Latest FY & $\$$ & $1,639,237$ & $\$$ & 75,933 & $\$ 53,170$ & $\$$ & 78,467 & $\$ 245,395$ & $\$ 1,035,737$ & $\$$ & $5,688,000$ & $\$ 177,995$ & $\$$ & 977,274 \\
\hline Revenue LTM & $\$$ & $1,675,822$ & $\$$ & 74,698 & $\$ 59,209$ & $\$$ & 770,659 & $\$ 242,217$ & $\$ 1,039,837$ & $\$$ & $5,829,000$ & $\$ 197,570$ & $\$$ & 888,794 \\
\hline 1 YR Growth & & -1.89 & & 14.44 & -8.58 & & -2.75 & 12.83 & -0.38 & & 7.89 & 68.98 & & -6.91 \\
\hline 2 Yr CAGR & & 2.47 & & 12.29 & 11.02 & & 21.68 & 16.3 & 5.65 & & 7.96 & 47.53 & & 7.43 \\
\hline EBITDA (LTM) & $\$$ & 237,019 & $\$$ & 6,816 & $\$ 12,997$ & $\$$ & 190,518 & $\$ 11,204$ & $\$ 459,497$ & $\$$ & $1,051,000$ & $\$ 53,353$ & $\$$ & 163,290 \\
\hline EBITDA \% Revenue & & 14.14 & & 9.12 & 21.95 & & 24.72 & 4.61 & 44.19 & & 18.03 & 27.00 & & 18.37 \\
\hline 1 YR Growth & & -28.17 & & 148.12 & -22.01 & & -11.27 & -142.22 & -6.11 & & 2.44 & 85.3 & & -37.28 \\
\hline 2 YrCAGR & & -5.35 & & 24.81 & 46.49 & & 43.77 & NA & 2.67 & & -1.24 & 65.62 & & NA \\
\hline EBIT (LTM) & $\$$ & 79,155 & $\$$ & 2,734 & $\$ 10,376$ & $\$$ & 24,825 & $\$ 2,383$ & $\$ 330,041$ & $\$$ & $(1,005,000)$ & $\$ 37,605$ & $\$$ & $(203,790)$ \\
\hline EBIT as \% Revenue & & 4.72 & & 3.66 & 17.52 & & 3.22 & 0.98 & 31.74 & & -17.24 & 19.03 & & -22.93 \\
\hline 1 YR Growth & & 10.12 & & NA & -31.61 & & -170.75 & NA & -5.57 & & NA & 53.71 & & NA \\
\hline 2 Yr CAGR & & NA & & 9.56 & 41.59 & & NA & NA & 0.32 & & NA & 44.78 & & NA \\
\hline Earnings (LTM) & $\$$ & 55,893 & $\$$ & 2,679 & 8,747 & $\$$ & 27,492 & $\$ 8,750$ & $\$ 309,823$ & $\$$ & $(922,000)$ & $\$ 31,871$ & $\$$ & $(154,433)$ \\
\hline Earnings as \% Revenue & & 3.34 & & 3.59 & 14.77 & & 3.57 & 3.6 & 29.8 & & -15.82 & 16.13 & & -17.38 \\
\hline 1 YR Growth & & 311.8 & & NA & -8.89 & & -343.6 & -159.1 & -12.11 & & NA & 40.86 & & NA \\
\hline 2 YrCAGR & & NA & & 6.7 & 26.25 & & NA & NA & 13.79 & & NA & 41.94 & & NA \\
\hline EPS Est. FY08 & & 0.06 & & 0.28 & 0.3 & & 0.93 & 0.31 & 1.63 & & -1.26 & 1.27 & & \\
\hline EPS Est. FY09 & & 0.25 & & 0.26 & 0.33 & & 0.98 & 0.3 & 1.81 & & -0.27 & 1.54 & & \\
\hline \multicolumn{15}{|l|}{ Invested Capital } \\
\hline Total Cash+Mkt Sec & $\$$ & 375,789 & $\$$ & 27,454 & $\$ 32,090$ & $\$$ & 267,224 & $\$ 50,776$ & $\$ 1,039,163$ & $\$$ & $1,584,000$ & $\$ 95,010$ & $\$$ & 336,000 \\
\hline Total Assets & $\$$ & $1,650,457$ & $\$$ & 71,976 & $\$ 91,339$ & & $1,776,621$ & $\$ 207,922$ & $\$ 2,543,439$ & $\$$ & $13,616,000$ & $\$ 285,838$ & $\$$ & $2,665,000$ \\
\hline Preferred Stock & $\$$ & - & $\$$ & - & $\$$ & $\$$ & - & $\$$ & $\$$ & $\$$ & - & $\$$ & $\$$ & - \\
\hline Market Equity & $\$$ & $1,512,641$ & $\$$ & 69,580 & $\$ 101,036$ & & $1,321,906$ & $\$ 61,907$ & $\$ 5,419,845$ & $\$$ & $3,082,213$ & $\$ 156,846$ & $\$$ & 446,219 \\
\hline Interest Bearing Debt & $\$$ & 152,295 & $\$$ & - & $\$$ & $\$$ & 18,574 & $\$$ & $\$ 1,150,227$ & $\$$ & $2,421,000$ & $\$ 3,288$ & $\$$ & 881,000 \\
\hline MV of Invested Capital & $\$$ & $1,289,147$ & $\$$ & 42,126 & $\$ 68,946$ & & $1,073,256$ & $\$ 11,131$ & $\$ 5,529,909$ & $\$$ & $3,919,213$ & $\$ 65,124$ & $\$$ & 991,219 \\
\hline Book Value of Equity & $\$$ & 878,062 & $\$$ & 58,657 & $\$ 80,934$ & & $1,620,355$ & $\$ 157,941$ & $\$ 1,052,528$ & $\$$ & $6,508,000$ & $\$ 238,909$ & $\$$ & $1,503,000$ \\
\hline Stock Price at $9 / 30 / 08$ & $\$$ & 3.39 & $\$$ & 4.49 & 3.60 & $\$$ & 7.76 & 2.31 & 29.43 & $\$$ & 4.05 & 4.68 & $\$$ & 0.12 \\
\hline Shares Outstanding & & 446,207 & & 15,497 & 28,066 & & 169,911 & 26,799 & 184,127 & & 791,040 & 33,514 & & $3,727,380$ \\
\hline
\end{tabular}

\begin{tabular}{|l|l|l|l|l}
\hline Note 1: Growth Rates use finacial data from FYE & Note 2: Winbond and Silicon Motion were converted from \$TW to US\$
\end{tabular} 
Appendix B-1

(continued)

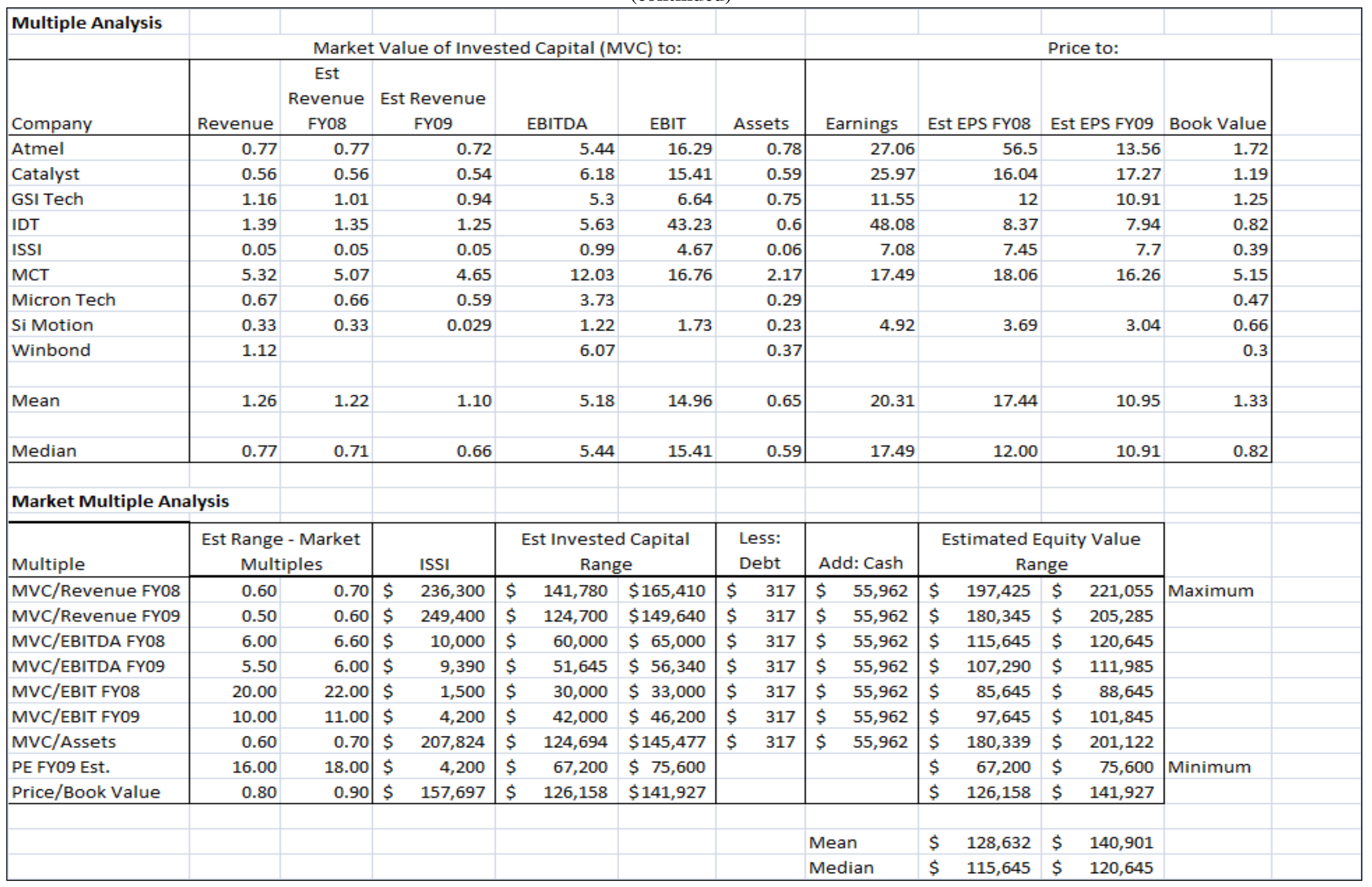


Appendix B-1

(continued)

\begin{tabular}{|c|c|c|c|c|c|}
\hline \\
\hline & & Estimated & & Implied & \\
\hline Multiple & & Value & ISSI & \multicolumn{2}{|c|}{ Market Multiple } \\
\hline MVC/Revenue FY08 & & $\$ \quad 76,560$ & $\$ 236,300$ & 0.32 & \\
\hline MVC/Revenue FY09 & & 76,560 & 249,400 & 0.31 & \\
\hline MVC/EBITDA FY08 & & 76,560 & 10,000 & 7.66 & \\
\hline MVC/EBITDA FYO9 & & 76,560 & 9,390 & 8.15 & \\
\hline MVC/EBIT FYO8 & & 76,560 & 1,500 & 51.04 & \\
\hline MVC/EBIT FYO9 & & 76,560 & 4,200 & 18.23 & \\
\hline MVC/Assets & & 76,560 & 207,824 & 0.37 & \\
\hline PE FY08 & & 132,205 & 7,700 & 17.17 & \\
\hline PE FY09 & & 132,205 & 4,200 & 31.48 & \\
\hline PE FY10 & & 132,205 & 8,580 & 15.41 & \\
\hline Price/BV & & 132,205 & 157,697 & 0.84 & \\
\hline \multicolumn{6}{|c|}{ Note: Market Value of Invested Capital (MVC) = Market Value of Equity plus Long Term Debt } \\
\hline \multirow[t]{4}{*}{ minus Cash. } & $\$ 132,205$ & & & & \\
\hline & 317 & & & & \\
\hline & 55,962 & & & & \\
\hline & $\$ 76,560$ & & & & \\
\hline
\end{tabular}


Appendix B-2

Weighted Average Cost of Capital ${ }^{14}$

\begin{tabular}{|c|c|c|c|c|c|c|c|c|c|}
\hline Company & $\begin{array}{c}\text { Total Debt } \\
\text { (000s) }\end{array}$ & $\begin{array}{c}\text { Stock } \\
\text { Price } \\
9 / 30 / 08 \\
\end{array}$ & Shares (000s) & $\begin{array}{c}\text { Preferred } \\
\text { Stock } \\
\end{array}$ & $\begin{array}{l}\text { Market Value } \\
\text { of Equity (000s) }\end{array}$ & $\begin{array}{l}\text { Debt as \% of } \\
\text { Total Capital }\end{array}$ & $\begin{array}{c}\text { Equity as } \% \text { of } \\
\text { Total Capital }\end{array}$ & $\begin{array}{l}\text { Levered } \\
\text { Beta (1) } \\
\end{array}$ & \\
\hline Atmel & 152,295 & $\$ 3.39$ & 446,207 & - & $\$ \quad 1,512,642$ & $9.15 \%$ & $90.85 \%$ & 2.92 & \\
\hline Catalyst & - & 4.49 & 15,497 & - & 69,582 & $0.00 \%$ & $100.00 \%$ & 1.08 & \\
\hline GSI Tech & - & 3.60 & 28,068 & - & 101,045 & $0.00 \%$ & $100.00 \%$ & 0.41 & \\
\hline IDT & 18,574 & 7.78 & 169,911 & - & $1,321,908$ & $1.39 \%$ & $98.61 \%$ & 1.88 & \\
\hline ISSI & - & 2.31 & 26,799 & - & 61,906 & $0.00 \%$ & $100.00 \%$ & 1.49 & \\
\hline Microchip Tech & $1,150,227$ & 29.43 & 184,127 & - & $5,418,858$ & $17.51 \%$ & $82.49 \%$ & 1.42 & \\
\hline Micron & $2,421,000$ & 4.05 & 761,040 & - & $3,082,212$ & $43.99 \%$ & $56.01 \%$ & 2.00 & \\
\hline Silicon Motion & 3,288 & 4.68 & 33,514 & - & 156,846 & $2.05 \%$ & $97.95 \%$ & 0.82 & \\
\hline \multirow[t]{2}{*}{ Windbond } & 881,000 & 0.12 & $3,727,380$ & - & 447,286 & $66.33 \%$ & $33.67 \%$ & 1.41 & \\
\hline & & & & \multicolumn{2}{|c|}{ SELECTED DATA } & $5.00 \%$ & $95.00 \%$ & 1.91 & \\
\hline COST OF DEBT & $\mathrm{Rd}=\mathrm{D}(1-\mathrm{t})$ & & & & & & & & \\
\hline \multirow[t]{3}{*}{ Where } & $D=$ & \multicolumn{3}{|c|}{$7.0 \%$ (Primate Rate $+2 \%$ ) (2) } & & & & & \\
\hline & $t=$ & \multicolumn{3}{|c|}{$40 \%$ Composite Federal Tax Rate } & & & & & \\
\hline & $\mathrm{Rd}=$ & \multirow[t]{2}{*}{$4.2 \%$} & & & & & & & \\
\hline COST OF EQUITY & $R e=R f+B(R p)+R s+R c$ & & & & & & & & \\
\hline \multirow[t]{6}{*}{ Where } & $\mathrm{Rf}=$ & \multicolumn{5}{|c|}{ 4.3\% (Risk Free Rate based on 30 Year Treasury Bond Rate) (2) } & & & \\
\hline & $B=$ & \multicolumn{2}{|c|}{1.91 (see above) } & & & & & & \\
\hline & $R p=$ & \multicolumn{3}{|c|}{$6.1 \%$ (Equity Risk Premium) (3) } & & & & & \\
\hline & Rs $=$ & \multicolumn{3}{|c|}{$3.7 \%$ (Small stock premium) (4) } & & & & & \\
\hline & $\mathrm{Rc}=$ & \multicolumn{3}{|c|}{$5.0 \%$ (Company Risk) (4) } & & & & & \\
\hline & $\mathrm{Re}=$ & \multicolumn{4}{|c|}{$24.6 \%$ (Expected return on Equity Capital) (4) } & & & & \\
\hline WACC & WACC $=\operatorname{Re}(e)+$ & $R d(d)$ & & & & & & & \\
\hline \multirow[t]{5}{*}{ Where } & $\mathrm{Re}=$ & $24.60 \%$ & & & & & & & \\
\hline & $e=$ & $95.00 \%$ & & & & & & & \\
\hline & $\mathrm{Rd}=$ & $4.20 \%$ & & & & & & & \\
\hline & $d=$ & $15.00 \%$ & & & & & & & \\
\hline & WACC $=$ & $24.00 \%$ & & & & & & & \\
\hline \multicolumn{3}{|c|}{ (1) Obtained from Reuters online. } & & & & & & & \\
\hline \multicolumn{10}{|c|}{ (2) Federal Reserve Statistical Release September 2008} \\
\hline \multicolumn{10}{|c|}{ (3) Ibbotson, R. G., \& Chen, P. (2003, Jan/Feb). Long run returns: Participating in the real economy. Financial Analysts Journal , 59 (1), pp. $88-98}$. \\
\hline \multicolumn{7}{|c|}{ (4) Morningstar, Inc. (2008). Stocks, Bonds, Bills, and Inflation Yearbook. Chicago: Mornin } & & & \\
\hline
\end{tabular}

${ }^{14}$ Prepared by Financial Strategies Consulting Group, LLC for ISSI. 


\section{ISSI FASB 142 Valuation Teaching Note}

Paula and the consultants elected to use two valuation methodologies; the Income Approach and The Market Approach to determine a fair value for the impairment analysis.

\section{The Income Approach}

The Income Approach utilizes a four-step methodology. First, future estimates are made of the revenue and earnings of the business as discussed previously. The financial projections are presented in the Appendices. Second, earnings before interest and taxes ("EBIT") is estimated and then tax effected to estimate debt-free net income. Debt-free net income is increased by depreciation, reduced by additions to fixed assets, and additions to working capital to estimate the net debt-free cash flow available for distribution to debt-holders and equity investors. Detailed information regarding working capital requirements can were estimated as was capital expenditures and depreciation/amortization. Third, the net debt-free cash flow stream is discounted to the present value by a required rate of return that reflects the risk profile of the Company. This required rate of return is calculated by estimating the Company's weighted average cost of capital ("WACC"). The WACC is computed by selecting market rates at the valuation date for debt and equity that are reflective of the risks associated with an investment in the subject industry as estimated by using comparable publicly-traded companies. The consultants computed the WACC to be $24.0 \%$, rounded, as detailed in Appendix B-2. This discount rate is what investors in ISSI would likely require to invest in the Company, as of the valuation date. Fourth, the terminal value is calculated to estimate the earnings of the business beyond the forecast period. The terminal value was calculated using a formula known as the Gordon Growth Model, whose formula is:

Terminal Value $=$ Debt-free cash flowterminal year $*(1+\mathrm{g}) /($ Discount Rate $-\mathrm{g})$

where "g" is the Company's long-term growth rate, which was estimated to equal $5.0 \%$ for the September 30, 2008 valuation date, which reflects both the long-term rate of inflation as well as ISSI's leading position in the industry. The terminal value is then discounted to its present value and added to the present value of the cash flows to estimate the Company's total invested capital value. Based on this analysis, the value of the discounted cash flows for ISSI equal \$56.6 million utilizing the Income Approach as of September 30, 2008.

\begin{tabular}{|c|c|c|c|c|c|c|c|c|c|}
\hline$\$ 000$ 's & & $9 / 30 / 2009$ & $9 / 30 / 2010$ & $9 / 30 / 2011$ & $9 / 30 / 2012$ & $9 / 30 / 2013$ & $\begin{array}{c}\text { Terminal } \\
\text { Value }\end{array}$ & Reference & \\
\hline Revenue & & 249,400 & 290,000 & 335,000 & 380,000 & 420,000 & & & \\
\hline COGS & & 192,000 & 221,800 & 252,900 & 285,000 & 310,800 & & & \\
\hline Gross Profit & & 57,400 & 68,200 & 82,100 & 95,000 & 109,200 & & & \\
\hline Operating Expenses & & 53,200 & 57,000 & 61,500 & 67,500 & 73,500 & & & \\
\hline Operating Income & & 4,200 & 11,200 & 20,600 & 27,500 & 35,700 & & & \\
\hline Taxes & & 1,680 & 4.48 & 8,240 & 11,000 & 14,280 & & & \\
\hline Net Income & & 2,520 & 6,720 & 12,360 & 16,500 & 21,420 & 21,420 & & \\
\hline Depreciation/Amortization & & 5,190 & 5,948 & 6,824 & 7,690 & 8,527 & 4,500 & Appendix A-1 & \\
\hline Capital Additions & & $(3,000)$ & $(3,500)$ & $(4,000)$ & $(4,500)$ & $(5,000)$ & $(5,000)$ & Appendix A-1 & \\
\hline Change in Working Capital & & $(5,986)$ & $(6,090)$ & $(6,750)$ & $(6,750)$ & $(6,000)$ & $(3,150)$ & $15 \%$ of Revenue & \\
\hline Free Cash Flow & & $(1,276)$ & 3,078 & 8,434 & 12,940 & 18,947 & 17,770 & & \\
\hline \multicolumn{2}{|l|}{ Terminal Value at $6 \%$ Growth } & & & & & & 98,203 & \multicolumn{2}{|c|}{ Gordon Growth Model } \\
\hline Present Value Factor & $24 \%$ & 0.898 & 0.7242 & 0.584 & 0.471 & 0.3798 & 0.3798 & & \\
\hline Present Value of Cash Flows & & $(1,146)$ & 2,229 & 4,925 & 6,095 & 7,196 & 37,297 & & \\
\hline Market Value of Invested Capita & & $\$ \quad 56,597$ & & & & & & & \\
\hline & & & & & & & & & \\
\hline Rounded & & 56,600 & & & & & & & \\
\hline
\end{tabular}




\section{Net Operating Loss Carryforwards}

As part of the analysis, it was determined that the Company had net operating loss carryforwards ("NOLs") available for future use. Management indicated that the Company had \$121.7 million in NOLS, respectively. For this analysis, the cumulative NOLs are projected to be fully utilized between FY 2009 and FY 2014, with no annual usage limitation. The yearly tax benefits arising from the NOLs were then adjusted to their present value and summed to equal $\$ 20.1$ million.

\begin{tabular}{|c|c|c|c|c|c|c|c|c|c|c|}
\hline \multicolumn{4}{|c|}{ Present Value of NOLs (\$000s) } & \multirow{2}{*}{\multicolumn{2}{|c|}{$9 / 30 / 2010$}} & \multirow{2}{*}{\multicolumn{2}{|c|}{$9 / 30 / 2011$}} & \multirow[b]{2}{*}{$9 / 30 / 2012$} & \multirow[b]{2}{*}{$9 / 30 / 2013$} & \multirow[b]{2}{*}{$9 / 30 / 2014$} \\
\hline & & & $9 / 30 / 2009$ & & & & & & & \\
\hline Available NOLs (1) & & $\$$ & $121,700.0$ & & & & & & & \\
\hline Operating Income & & $\$$ & $4,200.0$ & $\$$ & $11,200.0$ & $\$$ & $20,600.0$ & $\$ 27,500.0$ & $\$ 35,700.0$ & \\
\hline Beginning Balance & & $\$$ & $121,700.0$ & $\$$ & $117,500.0$ & $\$$ & $106,300.0$ & $\$ 85,700.0$ & $\$ 58,200.0$ & $\$ 22,500.0$ \\
\hline NOL Utilized & & $\$$ & $4,200.0$ & $\$$ & $11,200.0$ & $\$$ & $20,600.0$ & $\$ 27,500.0$ & $\$ 35,700.0$ & $\$ 22,500.0$ \\
\hline Tax Benefit (2) & $35 \%$ & $\$$ & $1,470.0$ & $\$$ & $3,920.0$ & $\$$ & $7,210.0$ & $\$ 9,625.0$ & $\$ 12,495.0$ & $\$ 7,875.0$ \\
\hline PV Factor & $24 \%$ & & 0.8980 & & 0.7242 & & 0.5840 & 0.4710 & 0.3798 & 0.3063 \\
\hline PV of NOL & & $\$$ & $1,320.1$ & $\$$ & $2,838.9$ & $\$$ & $4,210.6$ & $\$ 4,533.4$ & $\$ \quad 4,745.6$ & $\$ \quad 2,412.1$ \\
\hline TotalPV of NOLs & & & $20,060.7$ & & & & & & & \\
\hline & (1) Per 200 & $7 \mathrm{fi}$ & înancial sta & tem & hents & & & & & \\
\hline & (2) US Fed & era & I Tax Rate & & & & & & & \\
\hline
\end{tabular}

The Company's cash of $\$ 56.0$ million and NOL value of $\$ 20.1$ million were then added to the present value of cash flows of $\$ 56.6$ million, and debt of $\$ 317,259$ was subtracted to yield a $\$ 132.3$ million fair market value of equity.

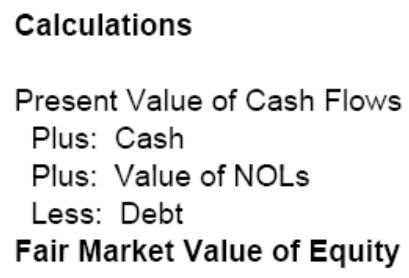

\section{Market Approach}

In applying the Market Approach, a four-step methodology was used. First, publicly-traded firms in the same or similar lines of business were identified. The comparability of each company listed is determined by reviewing their business description and financial history. The relevant financial data for each comparable company can be found in Appendix B-1.

Second, the market multiples are calculated for the guideline companies and are shown on page 13. Third, estimated market multiples are applied to the Company's actual and projected result. Fourth, implied market multiples are compared to the guideline company multiples as a further check. 


\section{MVIC/Revenue}

This ratio divides the aggregate market value of invested capital ("MVIC") by revenue. MVIC is defined as the sum of the market value of equity and interest-bearing debt less cash and marketable securities. This ratio allows for the quantification of the relationship between sales and total invested capital. The multiples for the guideline companies ranged from 0.05 to 5.32 with the mean and median equal to 1.26 and 0.77 , respectively. After analyzing analysts' estimates of the guideline companies' forecasted revenue, we calculated the MVIC/Revenue multiples based on estimated FY 2008 and FY 2009 revenue. The MVIC/Revenue multiples ranged from 0.05 to 5.07 with the mean and median equal to 1.22 and 0.71, respectively, based on estimated FY 2008 results, and ranged from 0.05 to 4.65 with the mean and median equal to 1.13 and 0.66, respectively, based on estimated FY 2009 results. Applying multiples between 0.6 and 0.7 to the Company's FY 2008 revenue yields an estimated invested capital value range between $\$ 141.8$ million and \$165.4 million. After subtracting debt and adding cash, the unit's estimated equity value ranged between \$197.4 million and \$221.1 million. Applying forward multiples between 0.5 and 0.6 to the Company's projected FY 2009 revenue yields on invested capital value range between \$124.7 million and \$149.6 million. After subtracting debt and adding cash, the unit's estimated equity value ranged between $\$ 180.3$ million and $\$ 205.3$ million. (Note: the multiples applied are below the guideline company mean and median multiples.)

\section{MVIC/EBITDA}

This ratio divides the aggregate MVIC by EBITDA. EBITDA is defined as earnings before interest, taxes, depreciation and amortization. This ratio shows a comparison of companies while minimizing differences between the companies' depreciation policies, financial leverage and taxes. The MVIC/EBITDA multiples for the guideline companies ranged from 0.99 to 12.03 with the mean and median equal to 5.18 and 5.44, respectively. Applying multiples between 6.0 and 6.5 to the Company's FY 2008 EBITDA yields an estimated invested capital value range between $\$ 60.0$ million and $\$ 65.0$ million. After subtracting debt and adding cash, the unit's estimated equity value ranged between $\$ 115.6$ million and $\$ 120.6$ million. Applying forward multiples between 5.5 and 6.0 to the Company's projected FY 2009 EBITDA yields an invested capital value range between $\$ 51.6$ million and $\$ 56.3$ million. After subtracting debt and adding cash, the unit's estimated equity value ranged between $\$ 107.3$ million and $\$ 112.0$ million.

\section{MVIC/EBIT}

This ratio divides the aggregate MVIC by EBIT. EBIT is defined as earnings before interest and taxes. This ratio shows a comparison of companies while minimizing differences between the companies' financial leverage and taxes. The positive MVIC/EBIT multiples for the guideline companies ranged from 1.73 to 43.23 with the mean and median equal to 14.96 and 15.41. Applying multiples between 20.0 and 22.0 to the Company's FY 2008 EBIT yields an estimated invested capital value range between $\$ 30.0$ million and $\$ 33.0$ million. After subtracting debt and adding cash, the unit's estimated equity value ranged between $\$ 85.6$ million and $\$ 88.6$ million. Applying forward multiples between 10.0 and 11.0 to the Company's projected FY 2009 EBIT yields on invested capital value range between $\$ 42.0$ million and $\$ 46.2$ million. After subtracting debt and adding cash, the unit's estimated equity value ranged between $\$ 97.6$ million and $\$ 101.8$ million. (Note: we applied high multiples to FY 2008 results given the low profitability and then used multiples at the lower end of the guideline company range based on FY 2009 results due to the higher expected profit margins.)

\section{MVIC/Assets}

This ratio divides the aggregate MVIC by the company's total assets and measures asset utilization. The multiples for the guideline companies range from 0.05 to 2.17 with a mean and median equal to 0.65 and 0.59 , respectively. Applying multiples between 0.6 and 0.7 to the Company's total assets yields an estimated invested capital value range between $\$ 124.7$ million and $\$ 145.5$ million. After subtracting debt and adding cash, the unit's estimated equity value ranged between $\$ 180.3$ million and $\$ 201.1$ million. 


\section{Price/Earnings}

This ratio divides the aggregate market value of equity by net earnings from recurring sources. The P/E multiples (excluding negative multiples) based on the LTM ranged from 4.92 to 48.08 with the mean and median equal to 20.31 and 17.49, respectively. After analyzing analysts' estimates of the guideline companies' forecasted earnings, we calculated the P/E multiples based on both estimated FY 2008 and FY 2009 earnings. The P/E multiples ranged from 3.69 to 56.50with the mean and median equal to 17.44 and 12.00 , respectively, based on estimated FY 2008results, and ranged between 3.04 to 17.27 with the mean and median equal to 10.95 and 10.91, respectively, based on estimated FY 2009 results. Applying forward multiples between 16.0 and 18.0 to projected FY 2009 earnings yields an estimated equity value range between $\$ 67.2$ million and $\$ 75.6$ million.

\section{Price/Book Value}

This ratio divides the aggregate market value of equity by the company's book value. P/BV multiples of the guideline companies range from 0.39 to 5.15 , with the mean and median equal to 1.33 and 0.82 , respectively. Applying multiples between 0.8 and 0.9 to the Company's book value yields an estimated equity value range between $\$ 126.2$ million and \$141.9 million.

\section{Conclusion of Value - Market Approach}

The following is a summary of the values estimated from the Market Approach:

MARKET APPROACH SUMMARY

September 30, 2008

(dollars in thousands)

\begin{tabular}{||c|c|c|c||}
\hline $\begin{array}{c}\text { Value } \\
\text { Indication }\end{array}$ & $\begin{array}{c}\text { Low End } \\
\text { Value Indication }\end{array}$ & & $\begin{array}{c}\text { High End } \\
\text { Value Indication }\end{array}$ \\
\hline Minimum & $\$ 67,200$ & - & $\$ 75,600$ \\
\hline Maximum & $\$ 197,425$ & - & $\$ 221,055$ \\
\hline Mean & $\$ 128,633$ & - & $\$ 140,901$ \\
\hline Median & $\$ 115,645$ & - & $\$ 120,645$ \\
\hline
\end{tabular}

Based upon the Market Approach, ISSI’s estimated equity value is between $\$ 115.6$ million and \$140.9 million.

\section{CONCLUSION}

Based on this analysis, the book value of ISSI as of September 30, 2008 equals approximately $\$ 157.7$ million. In determining if an impairment exists, it was estimated the Company's discounted after-tax cash flows at $\$ 56.6$, added cash of $\$ 56.0$ million, and NOL values of $\$ 20.1$ million and subtracted debt of $\$ 317,259$ to estimate the fair value of the equity to equal $\$ 132.3$ million. Then the fair value of the Company's equity was compared to the book value of equity. Since the fair value of the equity of $\$ 132.3$ million is less than the book value of equity of $\$ 157.7$ million, it was determined that, per FASB 142, an impairment exists. 
Impairment Conclusion

Valuation Conclusion

Book Value of Business Unit as of September 30, 2008

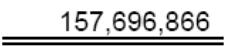

Fair Market Value of Equity

Difference

$25,391,729$

Impairment Applicable?

Yes

\section{AUTHOR INFORMATION}

John Zimmerman, Zayed University. After a successful career in industry as a senior executive in financial and entrepreneurial positions with recognized industry leaders such as General Electric Company. Caterpillar Corporation, Intel Corporation; and with venture capital financed emerging companies such as Level One Communications and iSuppli Corporation, Dr. Zimmerman secured his doctorate from Pepperdine University in Malibu, California. He has taught at Pepperdine University Graziadio School of Business, the University of Southern Nevada, and Zayed University in Abu Dhabi, UAE. His research interests include entrepreneurship and entrepreneurial finance.

\section{REFERENCES}

1. Consumer Electronics Association. (n.d.). 2008 Outlook. Retrieved June 20, 2008, from www.ce.org

2. Financial Standards Board. (2001). Retrieved August 15, 2010, from www.fasb.org

3. Financial Strategies Consulting, LLC. (2008). ISSI Valuation Report. San Jose, CA.

4. Ibbotson, R. G., \& Chen, P. (2003, Jan/Feb). Long run returns: Participating in the real economy. Financial Analysts Journal , 59 (1), pp. 88-98.

5. International Monetary Fund. (n.d.). Retrieved 30 2008, July, from www.imf.org

6. iSuppli Corporation. (2008). 2007 and 2008 Semiconductor Forecast Adjustments (Fact Sheet). El Segundo, CA.

7. Morningstar, Inc. (2008). Stocks, Bonds, Bills, and Inflation Yearbook. Chicago: Morningstar, Inc.

8. The Gartner Group. (2008, March 3). Gartner halves 2008 semiconductor outlook. Electronic News.

9. The McClean Report. (n.d.). IC Insights. Retrieved July 25, 2007, from www.icinsights.com

10. Zebrowski, P. (October). ISSI Financial Reports and Estimates. (J. Zimmerman, Interviewer) 


\section{NOTES}

